\title{
Design of a compensation mechanism for an active cardiac stabilizer based on an assembly of planar compliant mechanisms
}

\author{
L. Rubbert ${ }^{1, a}$, P. Renaud ${ }^{1}$, S. CAro ${ }^{2}$ And J. Gangloff ${ }^{1}$ \\ 1 ICube, CNRS, INSA de Strasbourg, Université de Strasbourg, France \\ 2 IRCCyN, CNRS, École Centrale de Nantes, France
}

Received 20 June 2013, Accepted 12 February 2014

\begin{abstract}
In this paper we present the design of a compact active cardiac stabilizer based on planar compliant mechanisms and piezoelectric actuators. Considering an assembly of planar manufactured structures helps to simplify the manufacturing process and may increase the compactness. Parallel architectures constitute interesting solutions for their intrinsic stiffness properties, but in a planar configuration parallel manipulators often exhibit kinematic singularities. Two design approaches for planar parallel compliant mechanisms are presented in this paper. One design approach consists in designing a passive compliant mechanism in a configuration close to the singularity by introducing some asymmetries during the manufacturing process. The second design approach consists in taking advantage of the singularities of parallel manipulators to obtain non-trivial solutions. The new proposed active stabilizer, composed of planar compliant mechanisms, is introduced and its performances are discussed.
\end{abstract}

Key words: Compliant mechanism / active cardiac stabilizer / mechanism design / parallel architecture / kinematic singularity

Résumé - Dans cet article, nous présentons la conception d'un mécanisme de compensation pour un stabilisateur cardiaque actif, composé de mécanismes compliants plans et d'actionneurs piézoélectriques. L'utilisation de mécanismes compliants plans facilite leur fabrication et favorise la compacité du dispositif. Recourir à des architectures parallèles pour la conception de mécanismes compliants permet généralement de disposer de propriétés de rigidité intéressantes. Cependant, la configuration plane des manipulateurs parallèles est souvent singulière. C'est pourquoi deux approches de conception de mécanismes compliants plans sont exploitées dans cet article. Une première approche consiste à explorer le voisinage de la singularité en introduisant des asymétries lors de la fabrication. Une seconde approche consiste à profiter de la singularité afin d'obtenir des architectures originales de mécanismes. Le dispositif complet à base de mécanismes compliants plans est présenté, et ses performances sont discutées.

Mots clés : Guidages flexibles / stabilisateur cardiaque actif / conception de mécanismes / architecture parallèle / singularité cinématique

\section{Introduction}

Surgical robotics helps to increase the surgeon's accuracy and limits the invasiveness of the surgery. The complexity of an operation room implies to design surgical devices that are as compact as possible and that can be easily sterilized. One interesting design approach is to combine compliant mechanisms, which have a monolithic structure, and piezoelectric actuators $[1,2]$. Based on this approach, a robotic device for minimally invasive coronary artery bypass grafting has been proposed

${ }^{a}$ Corresponding author: lennart.rubbert@epfl.ch previously in our laboratory [3]. It is composed of a shaft with two fingers in contact with the heart at one end, and an actuated compensation mechanism at the other end. The latest prototype, the Cardiolock II, is illustrated in Figure 1. This device successfully helps to increase the stabilization of the beating heart surface during the surgery but its compactness needs to be increased for an optimal integration in the operation room.

One possibility is to reduce the size of the compensation mechanism by considering an assembly of planar manufactured structures. This helps to simplify the manufacturing process and may increase the compactness. Lamina emergent mechanism (LEM) [4] and ortho-planar 


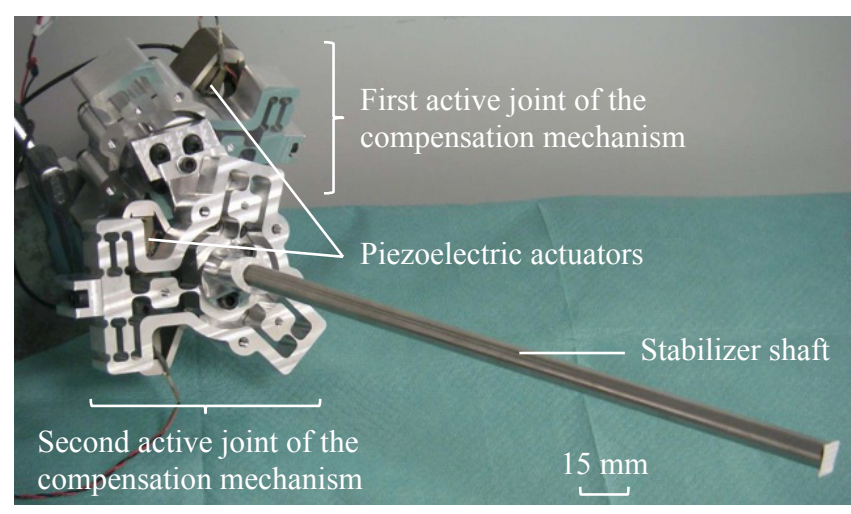

Fig. 1. The Cardiolock II.

mechanisms [5] are based on this principle. Compliant mechanisms can be designed with a so called kinematic approach, which consists in first selecting in the literature an architecture that answers to the kinematic requirements and then replacing each joint by a compliant joint using the pseudo-rigid body modeling [6]. In this paper, we follow this design approach. Therefore, in a first section, the kinematic scheme of the new compensation mechanism compatible with a planar decomposition is introduced. The joints are then designed as compliant structures, trying to use parallel compliant architectures for their intrinsic stiffness properties. In planar configurations, parallel manipulators often exhibit kinematic singularities [7]. To deal with these singularities, we consider in the first section two design approaches. One design approach consists in designing a passive compliant mechanism in a configuration close to the singularity by introducing some asymmetries during the manufacturing process. The second design approach consists in taking advantage of the singularities of parallel manipulators. The integration of the different components for the design of a compact device is presented in the second section. Finally, the active cardiac stabilizer composed of planar compliant mechanisms is presented and discussed in a third section.

\section{Design of the different components of the active cardiac stabilizer}

The stabilizer must be able to produce, similarly to the Cardiolock II device, displacements along the directions normal to the shaft axis to compensate for the flexibilities of the device and its mounting system. It is therefore a two degrees of freedom (DOF) mechanism, that will be actuated with piezoelectric actuators. One way to take advantage of the oblong geometry of the shaft is to decompose the compensation mechanism by distributing the mobilities into two planes, $\mathrm{P}_{1}$ and $\mathrm{P}_{2}$, close enough to provide a high displacement amplification ratio. Thus, we propose to use the kinematic scheme represented in Figure 2. The shaft is oriented with a spherical joint in $\mathrm{O}_{2}$ and actuated with two prismatic joints in $\mathrm{O}_{1}$. A coupling mechanism composed of a universal joint and a prismatic joint allows

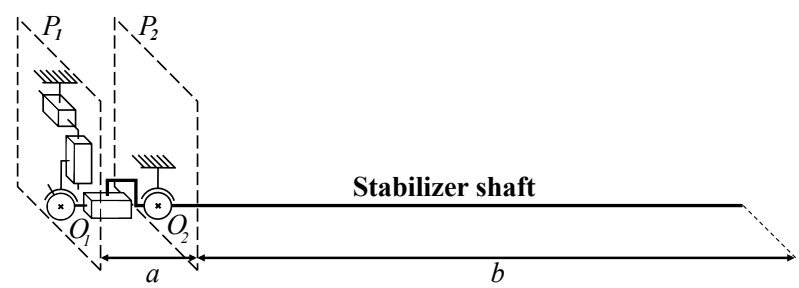

Fig. 2. Planar decomposition, kinematical scheme.

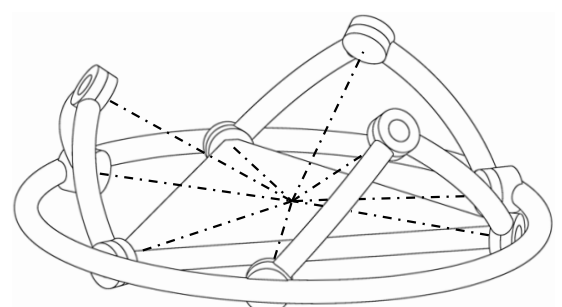

(a)

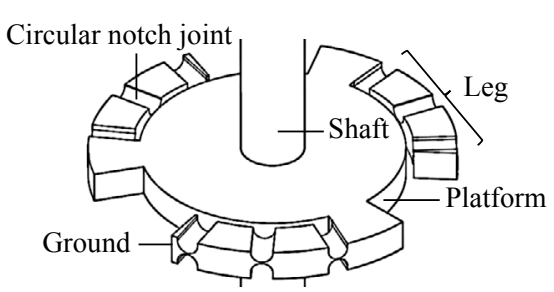

(b)

Fig. 3. Planar compliant orientation mechanism obtained from a 3-RRR spherical mechanism. (a) 3-RRR spherical mechanism. (b) 3-RRR compliant orientation mechanism.

to link the orientation and actuation mechanisms. Given the application requirements, the distance $\mathrm{O}_{1} \mathrm{O}_{2}$ must be less or equal to $12 \mathrm{~mm}$. This constrains the design of the device components presented in the following part of the section.

\subsection{Vicinity of singularities for the design of a planar compliant orientation mechanism}

For the design of the passive compliant spherical joint in $\mathrm{P}_{2}$, we choose the architecture of the 3 -RRR parallel spherical manipulator proposed by [8]. In the planar configuration, this manipulator is however in a singular configuration of type 1 and type 2 [9]. One approach used for LEMs in [10] is to design the 3-RRR compliant mechanism in the planar configuration and then to extract it to its working configuration. The extraction process seems complex, since the mechanism needs to be precisely positioned in a configuration that is close to the planar configuration. We prefer to design a passive compliant mechanism in the configuration illustrated in Figure $3 \mathrm{a}$ but close to the singularity by manufacturing the second revolute joints of each leg slightly out of the mid-plane. The modeling and the pre-dimensioning of the 3-RRR compliant orientation mechanism, illustrated in Figure 3b, have been addressed in [11]. 


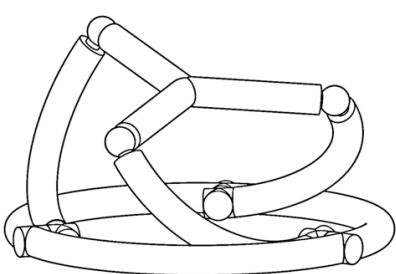

(a)

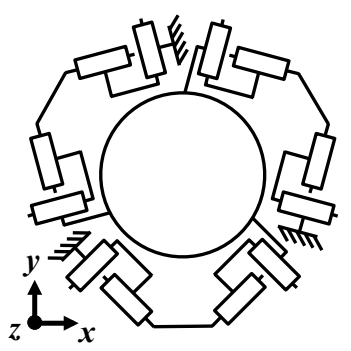

(b)

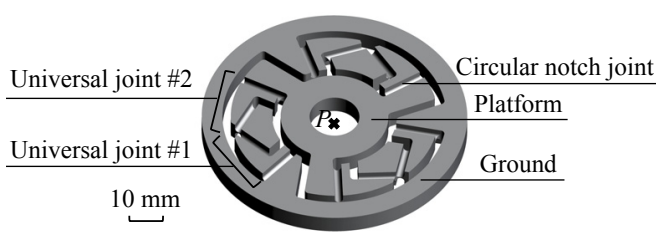

(c)

Fig. 4. Compliant coupling joint with RRP mobilities obtained from the singularity analysis of a 3-US parallel manipulator. (a) 3-US mechanism. (b) 3-UU mechanism. (c) 3-UU compliant coupling mechanism.

\subsection{Parallel singularities for the design of a planar compliant mechanism with RRP mobilities}

For the design of the coupling mechanism in $P_{1}$, we need two rotations (RR) around the in-plane axes in $\mathrm{O}_{2}$ and one translation $(\mathrm{P})$ along the shaft axis. We therefore choose the architecture of the 3-US parallel manipulator, illustrated in Figure 4a, considered for foldable mechanisms in [12]. In the planar configuration, the 3 -US parallel manipulator has the desired mobilities but is in a singular configuration of type 2 . It means that the endeffector of the manipulator loses stiffness while its actuators are blocked. As compliant mechanisms only work around a given configuration, this loss of stiffness can be used to produce the required mobilities. The use of this kind of singularity has been considered for the design of 1-DOF compliant mechanism in $[13,14]$. Here we want to obtain three mobilities from the singular configuration of the 3-US manipulator. The singularity analysis of the 3-US manipulator has been performed in [15] and has shown that a 3-UU planar mechanism, illustrated in Figure $4 \mathrm{~b}$, has locally the desired RRP mobilities. Finite element analysis (FEA) shows that in-plane stiffnesses are 200 times greater than the out-of-plane stiffness, and the torsional stiffness is 400 times greater than the other rotational stiffnesses. The equivalent 3-UU compliant mechanism, illustrated in Figure 4c, exhibits RRP mobilities.

\subsection{Selection of an actuation mechanism}

The coupling mechanism must be integrated in one of the two planes. As the 3-RRR compliant orientation mechanism will be integrated in the first plane, the coupling mechanism will be integrated in the second one. Therefore, the coupling mechanism will be integrated in the actuation mechanism. Among the 2-DOF planar parallel mechanism proposed in the literature, the 2PRR-RR mechanism proposed by [16] is particularly relevant: it has a planar geometry and it can integrate the coupling mechanism in its platform. As it will be actuated by piezoelectric actuators, it will only work around a given configuration. Considering $q_{1}$ and $q_{2}$ the actuators positions and $x$ and $y$ the cartesian coordinates of the platform point $\mathrm{O}_{2}$, the configuration illustrated in Figure 5 allows,

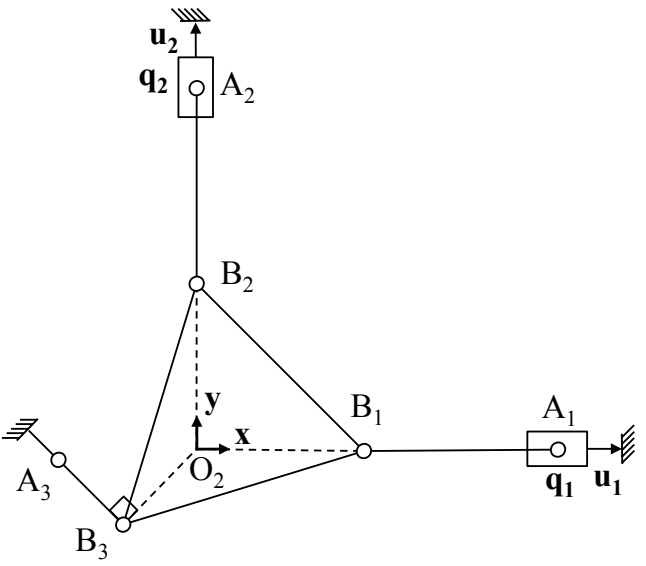

Fig. 5. Chosen configuration for the 2-PRR 1-RR actuation mechanism.

locally for small displacements, a decoupling and a direct transmission of the actuators to $\mathrm{O}_{2}$ as shown by the Jacobian matrix in equation (1). As the ratio between the actuators displacements $\delta x, \delta y$ and the distance $\mathrm{B}_{3} \mathrm{O}_{2}$ is high, the induced rotation $\delta \theta$ of the platform remains of small amplitude, and is therefore not a concern for the application.

$$
\left(\begin{array}{l}
\delta x \\
\delta y \\
\delta \theta
\end{array}\right)=\left(\begin{array}{cc}
1 & 0 \\
0 & 1 \\
\frac{-\sqrt{2}}{2 B_{3} O_{2}} & \frac{\sqrt{2}}{2 B_{3} O_{2}}
\end{array}\right)\left(\begin{array}{l}
\delta q_{1} \\
\delta q_{2}
\end{array}\right)
$$

\section{Integration of the three mechanisms in the two planes}

The different components of the compensation mechanism must be integrated in only two planes. In the first plane, only the orientation mechanism must be integrated in contrary to the second plane which contains the coupling mechanism and the actuation mechanism. Therefore, we propose, in addition to the 3-RRR compliant mechanism, to integrate the two piezoelectric actuators in the first plane. Since the piezoelectric actuators need obviously to be linked to the actuation mechanism, the two compliant prismatic joints of the actuation mechanism 


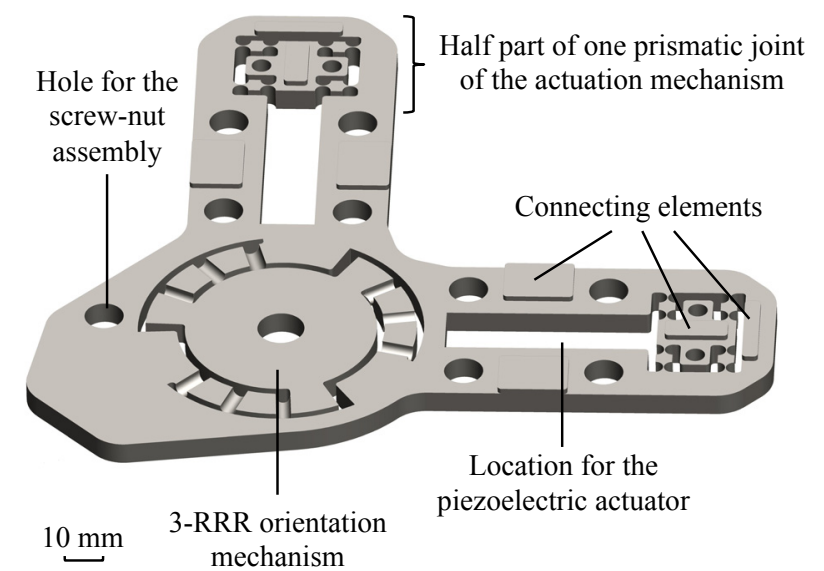

(a)

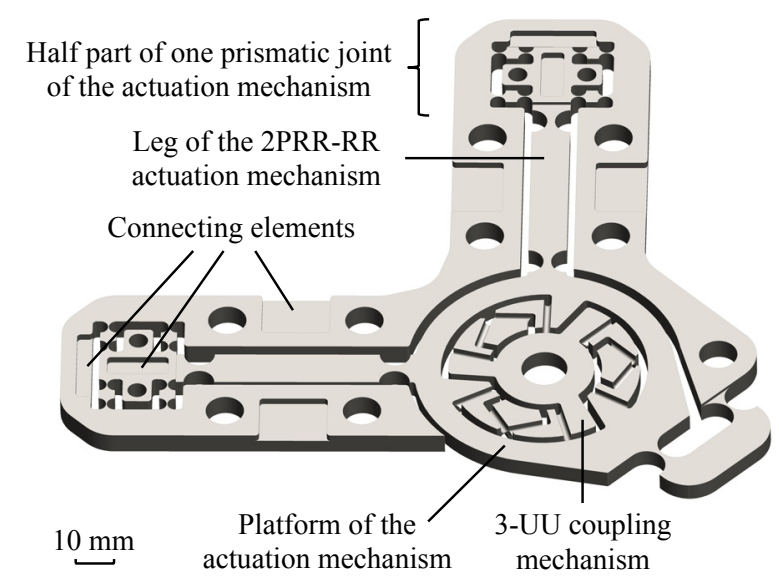

(b)

Fig. 6. Integration of the planar compliant mechanisms. (a) First plane of the compensation mechanism. (b) Second plane of the compensation mechanism.

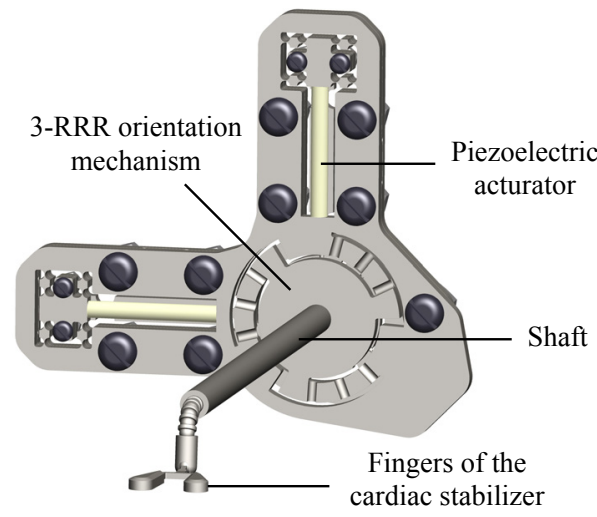

(a)

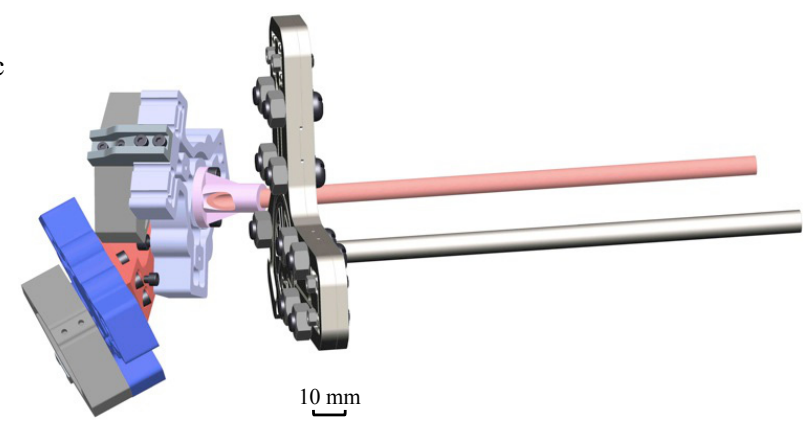

(b)

Fig. 7. Compensation mechanism based on planar compliant mechanisms. (a) Front view of the final device. (b) Size comparison between the Cardiolock II and the new device.

are decomposed in two parts designed in the two planes with connecting elements in order to transmit the actuator forces from the first plane to the second plane. This is illustrated in Figure 6a, where the first machined plane is illustrated. It contains the 3-RRR compliant mechanism for the shaft orientation, the two locations for the piezoelectric actuators and the two half compliant prismatic joints for the force transmission with the second plane. In the second machined plane, illustrated in Figure 6b, we can find the actuation mechanism with the embedded 3-UU compliant coupling mechanism. The two planes are assembled and maintained in position with dedicated connecting elements, screws and nuts.

\section{Results and discussion}

The assembled device with its piezoelectric actuators is illustrated in Figure 7a. It is first interesting to outline that the device is four times more compact than the previous one as illustrated in Figure 7b. After a simple trial and error pre-dimensioning of the whole mechanism using FEA, we furthermore already obtain interesting performances. The presented design allows us to produce a displacement of $0.7 \mathrm{~mm}$ which is sufficient to compensate for the flexibilities of the device and the mounting system, according to [3].

\section{Conclusions}

In this paper we have presented the design of a $2-\mathrm{DOF}$ compensation mechanism for an active cardiac stabilizer based on planar compliant mechanisms. To maximize the device compactness, planar structures are of interest. For parallel architectures, it may conduct to singular configurations. Two design approaches are thus considered in this paper. Singularities are once avoided by introducing asymmetry in the manufacturing of the compliant structure. We have then taken advantage of the singularities 
to design a passive parallel architecture with interesting stiffness properties. The new proposed device has been presented, with encouraging performances and a significant improvement in the compactness as expected. Global modeling of the device needs now to be achieved in order to optimize the level of performances, as well as further experimental work.

\section{References}

[1] D.Y. Choi, C.N. Riviere, Flexure-based manipulator for active handled microsurgical instrument, in Proceedings of the 27th Annual International Conference of the IEEE Engineering in Medicine and Biology Society, 2005, pp. $2325-2328$

[2] S. Kota, K.-J. Lu, Z. Kreiner, B. Trease, J. Arenas, J. Geiger, Design and application of compliant mechanisms for surgical tools, J. Biomech. Eng. 127 (2005) 981-989

[3] W. Bachta, P. Renaud, E. Laroche, J. Gangloff, The cardiolock project: Design of an active cardiac stabilizer for cardiac surgery, ASME J Mech. Des. 133 (2011) 1-10

[4] J.O. Jacobsen, B.G. Winder, L.L. Howell, S.P. Magleby, Lamina emergent mechanisms and their basic elements, J Mech. Robot. 2 (2010) 011003

[5] J.J. Parise, L.L. Howell, S.P. Magleby, Ortho-planar linear-motion springs, Mech. Mach. Theory 36 (2001) 1281-1299

[6] L.L. Howell, Compliant Mechanism, Wiley-IEEE (2001)

[7] C. Gosselin, J. Angeles, Singularity analysis of closedloop kinematic chains, IEEE Trans. Robot. Automat. 6 (1990) 281-290

[8] C.M. Gosselin, E. St-Pierre, Development and experimentation of a fast 3-dof camera-orienting device, Int. J. Rob. Res. 16 (1997) 619-630
[9] I.A. Bonev, C.M. Gosselin, Analytical determination of the workspace of symmetrical spherical parallel mechanisms, IEEE Trans. Robot. 22 (2006) 1011-1017

[10] J.O. Jacobsen, G. Chen, L.L. Howell, S.P. Magleby, Lamina emergent torsional (let) joint, Mech. Mach. Theory 44 (2009) 2098-2109

[11] L. Rubbert, P. Renaud, J. Gangloff, Design and optimization for a cardiac active stabilizer based on planar parallel compliant mechanism, In Proceedings of the ASME 2012 11th Biennial Conference on Engineering Systems Design and Analysis, 2012

[12] Y. Qin, J.S. Dai, Forward displacement analysis of two foldable 3us parallel mechanisms, In: Jian S Dai, Matteo Zoppi, and Xianwen Kong (eds.), Advances in Reconfigurable Mechanisms and Robots I, Springer London, 2012, pp. 805-814.

[13] D. Chapuis, R. Gassert, L. Sache, E. Burdet, H. Bleuler, Design of a simple mri/fmri compatible force/torque sensor, in Proceedings of the IEEE/RSJ International Conference on Intelligent Robots and Systems, 3 (2004) 2593-2599

[14] P. Renaud, M. de Mathelin, Kinematic analysis for a novel design of mri-compatible torque sensor, in Proceedings of the IEEE/RSJ International Conference on Intelligent Robots and Systems, 2009, pp. 2640-2646

[15] L. Rubbert, S. Caro, P. Renaud, J. Gangloff, A planar compliant mechanism with rrp mobilities based on the singularity analysis of a 3-us parallel mechanism, in Latest Advances in Robot Kinematics, Advances in Robot Kinematics series, Dordrecht Springer, 2012, pp. $381-388$

[16] C.M. Gosselin, Kinematische und statische analyse eines ebenen parallelen manipulators mit dem freiheitsgrad zwei: Kinematic and static analysis of a planar twodegree-of-freedom parallel manipulator, Mech. Mach. Theory 31 (1996) 149-160 